\title{
INBODY: Instant Photogrammetric 3D Body Scanner
}

\author{
Stanislao GRAZIOSO*1, Mario SELVAGGIO ${ }^{1,}$ Giuseppe DI GIRONIMO', Roberto RUGGIERO² \\ ${ }^{1}$ Department of Industrial Engineering, University of Naples "Federico II", Naples (NA), Italy; \\ ${ }^{2}$ Ortopedia Ruggiero srl, Cardito (NA), Italy
}

DOI: 10.15221/16.296 http://dx.doi.org/10.15221/16.296

\begin{abstract}
The digital manufacturing processes initially developed in rapid prototyping centers are nowadays appearing not only in industry, but also in medicine. In particular, in orthopedics, the manufacturing of orthoses is following this trend. The digital manufacturing process of orthoses requires a scanning device for the three-dimensional (3D) digitization of the human body part whose deformity needs to be corrected. However, the slowness of acquisition phase of the recent body scanners may constitute a key issue of the overall process, especially for patients with mobility impairments.

This work aims at presenting INBODY, an instant photogrammetric 3D full body scanner. The motivation behind it is to speed up the acquisition phase of $3 D$ human models, up to make it instantaneous. INBODY provides several features of interests in 3D body scanning technologies: (i) instant acquisition of the human body model; (ii) precision and accuracy of the resulting 3D model comparable with laser systems; (iii) affordable costs.

INBODY is built upon a modular and distributed architecture: in this paper we highlight its key concepts and illustrate its potential through a case study, the real time acquisition and the 3D reconstruction of a human full body and a human torso, used for the digital manufacturing process of orthoses.

Moreover, INBODY can be used also in other fields, whenever body measurements and 3D human models are needed (sport, medicine, fashion, ...).
\end{abstract}

Keywords: 3d body scanning, instant 3d body scanner, photogrammetry, human body measurements, orthoses.

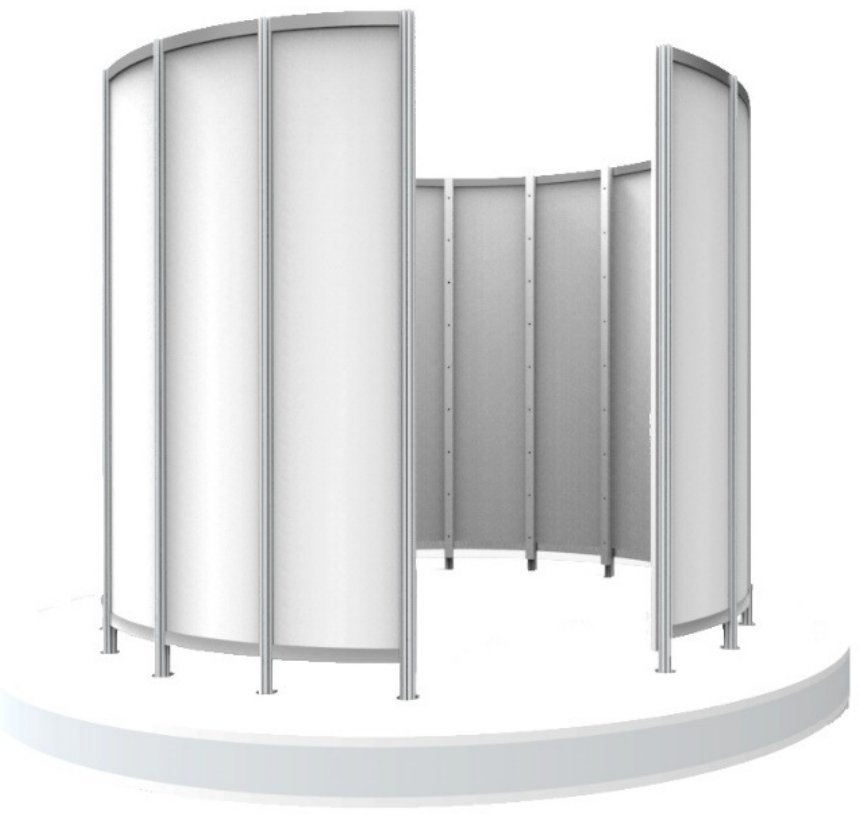

Fig. 1. INBODY

\section{Introduction}

As the development of digital technologies is growing up, manufacturing processes are moving towards the processes referred as digital manufacturing [1]. Medical related fields are following this trend as well [2], with applications related to: design and manufacturing of bio-models, surgical aid tools and implants, scaffolds for tissue engineering or development of surgical training models and medical devices [3].

* stanislao.grazioso@unina.it; +39-340-331-8138; +1-301-412-5810; 
Three-dimensional geometric data of anatomical structures, which are constructed with Reverse Engineering (RE) techniques, are used as input for medical Rapid Prototyping (RP) methods such as Additive Manufacturing (AM) or classic CNC Subtractive Manufacturing, the Computer Aided Design/Manufacturing (CAD/CAM). According to Hieu et. al [3], four main steps are required for medical RP:

1) Data acquisition;

2) Data registration and processing;

3) Geometrical modeling and design;

4) Medical application development

The main scenario behind this work is orthopedics, being interested in the RP of orthoses. An orthosis is "an externally applied device used to modify the structural and functional characteristics of the neuromuscular and skeletal system" [4].

Orthoses digital manufacturing process requires the following phases: 1) data acquisition, registration and processing for obtaining a positive of the patient's body part where the orthosis will be applied; 2) design, consisting in modifying and correcting the positive patient's 3D model, made by an orthopedic technician; 3) CNC milling process for obtaining a positive in polyurethane which reproduces the patient's 3D model modified by the technician; 4) building of the orthosis by liquefying, under vacuum conditions, an artificial resin around the positive model designed in 2) and manufactured in 3).

Data acquisition, registration and processing require modern three-dimensional body scanning systems. A strict requirement for these systems is the instant acquisition of the patient's body, especially for patients with mobility impairments. Moreover, for the design of orthoses modelled on the patient's exact body shape, the requirement of accuracy for such systems is of great importance as well.

As result, a three-dimensional body scanning system suitable for orthopedics should be characterized by: (i) instant acquisition of the human body model; (ii) precision and accuracy of the 3D models comparable with the best 3D scanning technologies; (iii) affordable costs to allow orthopedics in using it in their productive cycles.

In this paper we present INBODY, a 3D full body scanner system developed by the authors for orthopedics applications. The resulting body scanner allows the instant acquisition of the full human body model by taking synchronous images from different points of view and the 3D reconstruction of the human body surface with photogrammetric techniques. The 3D models obtained with INBODY present good characteristics if compared with models digitized with laser systems, which are considered the best scanning technologies in terms of accuracy and precision [5]. The problems of using laser system in orthopedics reside in their slow acquisition of the patient's body and their high cost.

Photogrammetry is the technology of obtaining three-dimensional objects from two-dimensional photographic images consisting in two steps: acquisition and photo elaboration (points cloud and surface reconstruction). The most used methods for points cloud generation are based on the Scale Invariant Feature Transform (SIFT) algorithm proposed by Lowe in [6], while the surface interpolation methods are generally based on Poisson reconstruction or ball pivoting algorithms ([7], [8]).

A comprehensive study about photogrammetry, and in general about multiple view geometry in computer vision can be found in [9].

The problems associated with the photogrammetry are listed as follows: 1) for a proper 3D reconstruction of an object, the scene should be fixed, i.e. the multiple photos should be taken in the same temporal instant; 2) the scene has to present a right amount of light (however adjustable modifying the ISO of the cameras); 3) the cameras should be placed in the correct space position to both avoid occlusions and guarantee a good key-points correspondence between more images, a fundamental aspect for the SIFT algorithm.

INBODY has been developed taking into consideration all these aspects: many theoretical considerations and experiments lead to the prototype shown in Fig. 3.

The remaining part of this paper is organized as follows. In Section 2 we describe the distributed architecture of INBODY, focusing on its hardware and software aspects and we present the developed working prototype. Section 3 is related to the benchmark tests which validate INBODY as a possible solution for instant acquisition of human models, after comparing the resulting 3D models with lasers reconstructed models.

The strength of this body scanner is that it can be used in many application fields wherein human body digitization can be useful (sport, medicine, fashion and beauty, cosmetic and dermatology). 


\section{INBODY overview}

INBODY is built upon a modular and distributed architecture, briefly described as follows.

\subsection{Hardware}

INBODY it is composed by a series of micro-cameras each one connected with its own micro-controller. All micro-controllers are connected between them and with a remote management controller via ethernet. Fig. 2 shows the conceptual hardware setup.

\subsection{Software}

INBODY control and management software is built upon a client multiple server architecture [10]: the client is represented by the remote management controller, while each micro-controller connected with its proper micro-camera represents a server, since it provides a series of services.

Each server has to guarantee a series of services, each one represented by a thread: taking and transferring pictures, shutting down each micro-controller.

A multi-thread software architecture [11] provides the synchronization of threads on each server: when the remote management controller sends the signal corresponding to one of the three threads, all servers provide the corresponding service instantaneously, without delay between them.

Moreover, the client provides the 3D reconstruction starting from the pictures. The reader can refer to Fig. 2 for the INBODY software architecture, based on the client - server paradigm.

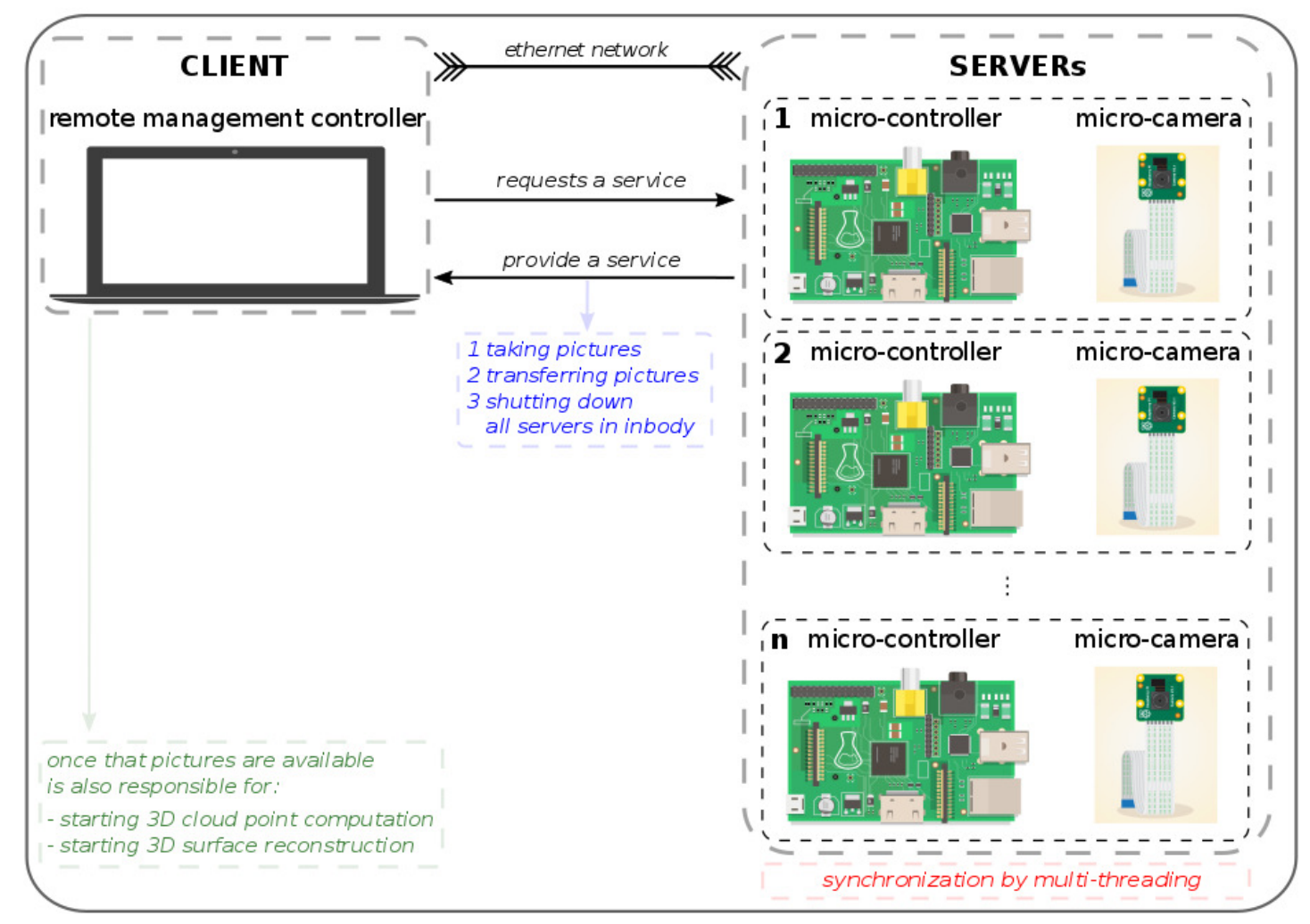

Fig. 2. INBODY network setup and software architecture

\subsection{Prototype}

The number of the cameras for obtaining a proper reconstruction of the full human body, as well as their disposition in space have been obtained by statistical experiments.

The mechanical structure of INBODY has been designed taking into account both functional and aesthetics requirements. Also the mechanical design is based on a modular architecture: starting from the bottom, plastic covers guarantee the supports of the micro-cameras. To position the micro-cameras in the correct space position for a proper 3D reconstruction, the assembly constituted by a camera with its cover is mounted on aluminium poles by means of lead screws. Each vertical profile is mounted on an iron circular base. To guarantee stability of the cylindrical structure, rectangular calendered profiles are connected to the vertical poles by means of special clamps. Fig. 3 shows the built prototype, corresponding to the render of Fig. 1. 


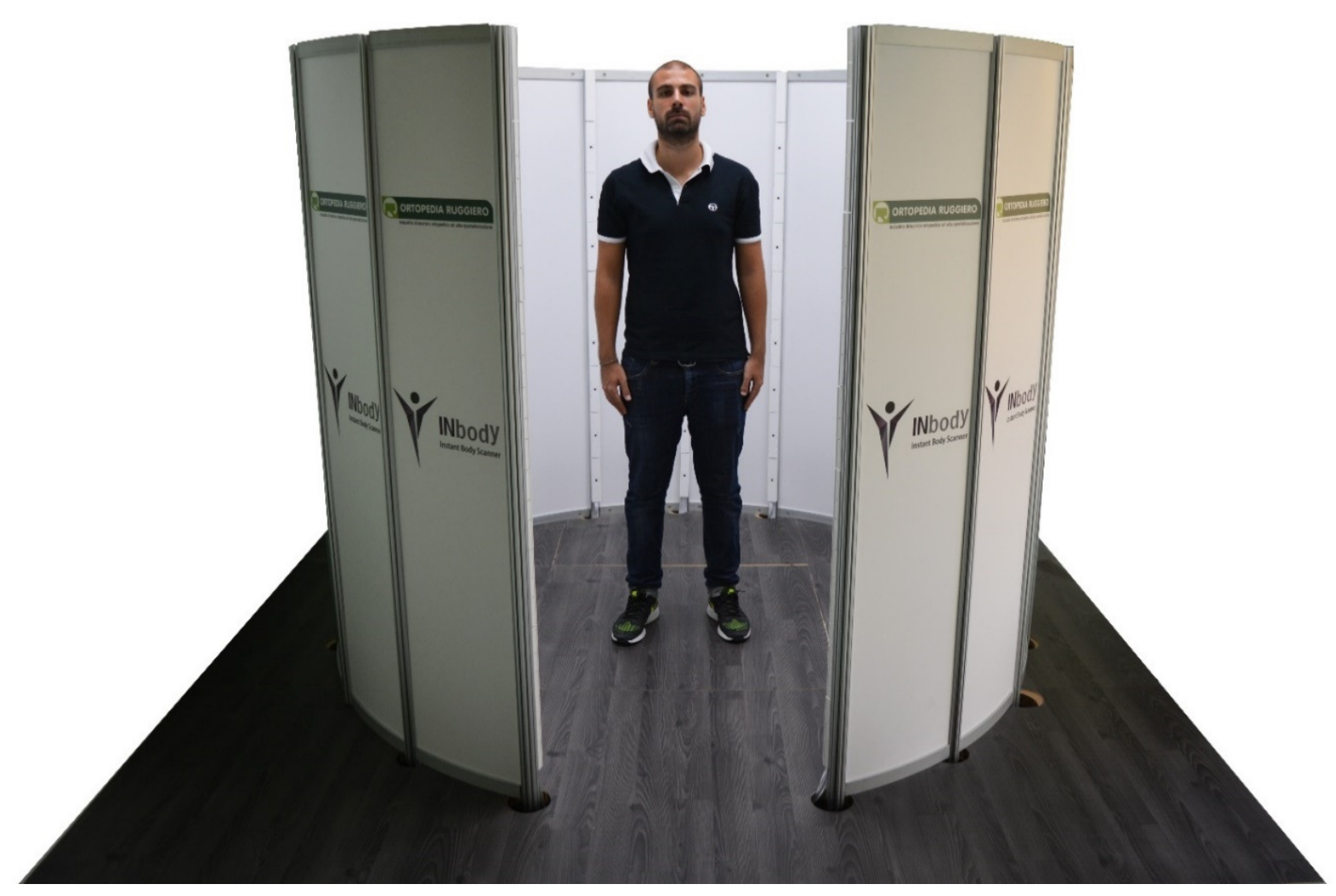

Fig. 3. INBODY prototype

\section{Benchmark tests}

INBODY has been tested with a lot of experiments, to test both the synchronization of the threads (in particular for the acquisition phase) and the 3D reconstruction of the human model.

In these experiments INBODY client has been represented by an Intel ${ }^{\circledR}$ Core ${ }^{\mathrm{TM}}$ i7-4910MQ CPU (quad-core $2.50 \mathrm{GHz}$, Turbo $3.50 \mathrm{GHz}$ ), $32 \mathrm{~Gb}$ RAM 1600MHz DDR3L, NVIDIA®Quadro®K2100M w/2GB GDDR5 VGA machine, running Ubuntu 1464 bits. Note that INBODY client may be also represented by a standard cheap microcontroller, if the images are processed on a remote workstation.

Agisoft PhotoScan [12] has been used for the points cloud generation and the 3D surface reconstruction.

Fig. 4 shows different 3D models obtained with INBODY. The first image proves the use of INBODY as a texture generator, the second one proves its use in digitizing difficult surfaces like the human skin without using markers. The third one is a torso 3D model, used for designing ad-hoc orthosis. Table 1 reports the computational time in generating the cloud point for the showed torso and the full body models, starting respectively with 50 and 120 images ( $2592 \times 1944$ pixels).
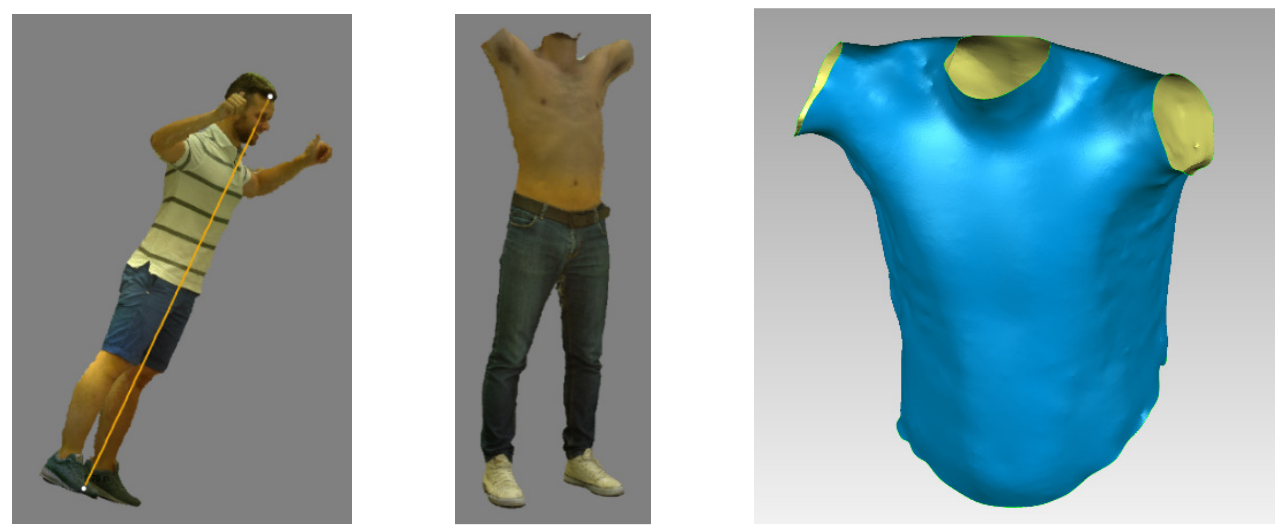

Fig. 4. 3D models obtained using INBODY. From the left: full body acquisition for texture and measurements objectives; full body acquisition of a difficult surface like the human skin; torso geometric model. 


\subsection{Torso model and comparisons}

In this section we deal with the torso human model reconstruction, since the main application for INBODY is the manufacturing of orthoses.

Fig. 5 shows a comparison between the torso model reconstructed with INBODY (the one showed also in Fig. 4) and the same torso model reconstructed with a manual laser scanner, Polhemus FastSCAN SCORPION [13]. We measure the deviations of the INBODY model (the grey one) with respect to the laser one, assumed as reference, thus presenting the color map. The deviations in the central part of the torso (the most important for orthoses design) are in the range $[-5 ;+5] \mathrm{mm}$. The average 3D deviation is of $[-11 ;+4] \mathrm{mm}$. INBODY has been validated also with static objects (mannequin) and the differences with the mentioned laser system result in an average 3D deviation of $[-3 ;+2] \mathrm{mm}$ (see Fig. 6). Even if the strength of this scanning system relays on digitalizing non-static objects, this benchmark test validates INBODY accuracy, which is better to verify with not moving objects.

However, the 3D deviations in both Fig. 5 and 6, especially the maximum and the minimum values (in the extreme part of the models) seems high since the alignment of the two models in the figures does not match perfectly. For example, if we consider Fig. 5, when the frontal part presents a negative deviation, the corresponding back part presents a positive deviation. This derives from the fact that it is really difficult to fit the models in the best way, being the three-dimensional surfaces.

The fact that the average 3D deviations, which also include the maximum and minimum values, are respectively $[-11 ;+4] \mathrm{mm}$ and $[-3 ;+2] \mathrm{mm}$ testify the good accuracy of INBODY.

Table 1. Computational time required for the generation of the points clouds

\begin{tabular}{|c|c|}
\hline Model & Computational time [minutes] \\
\hline Torso & 3.5 \\
\hline Full body & 9 \\
\hline
\end{tabular}
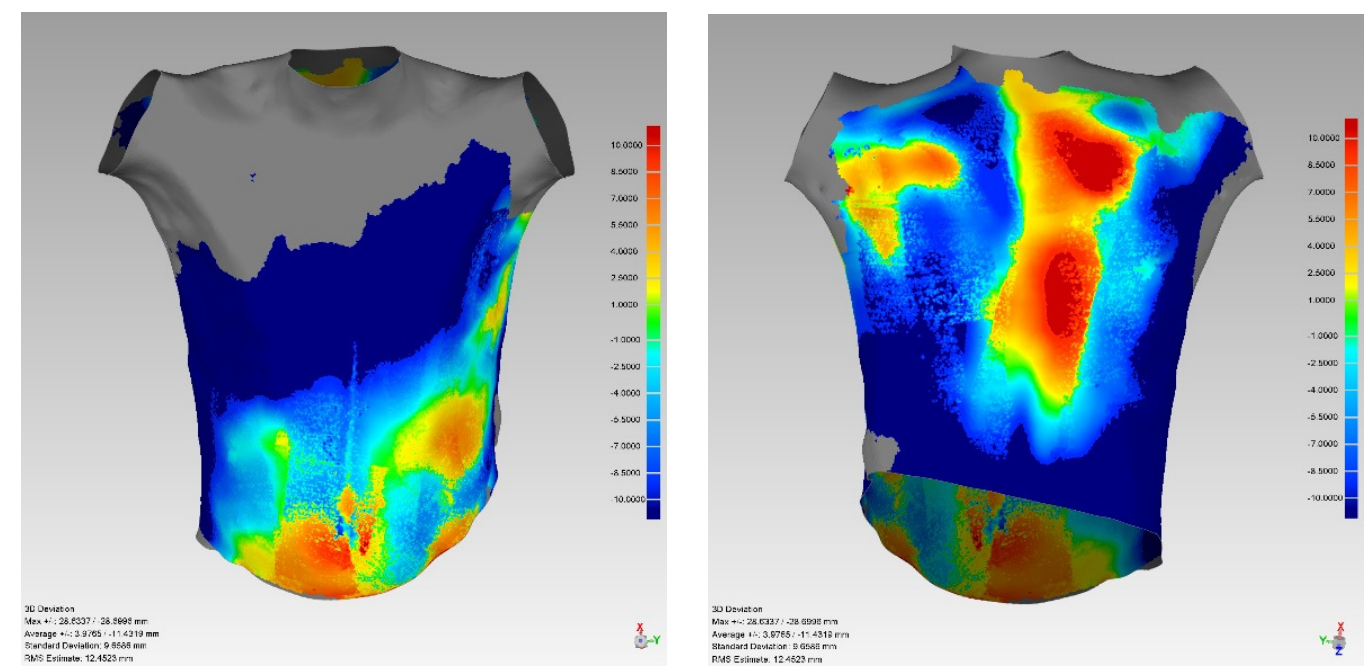

Fig. 5. INBODY 3D reconstruction compared with a laser reconstruction (assumed as reference) for a human torso. Note that the errors, in [mm], are measured on the laser reconstruction, the one which presents problems in the upper part of the model.
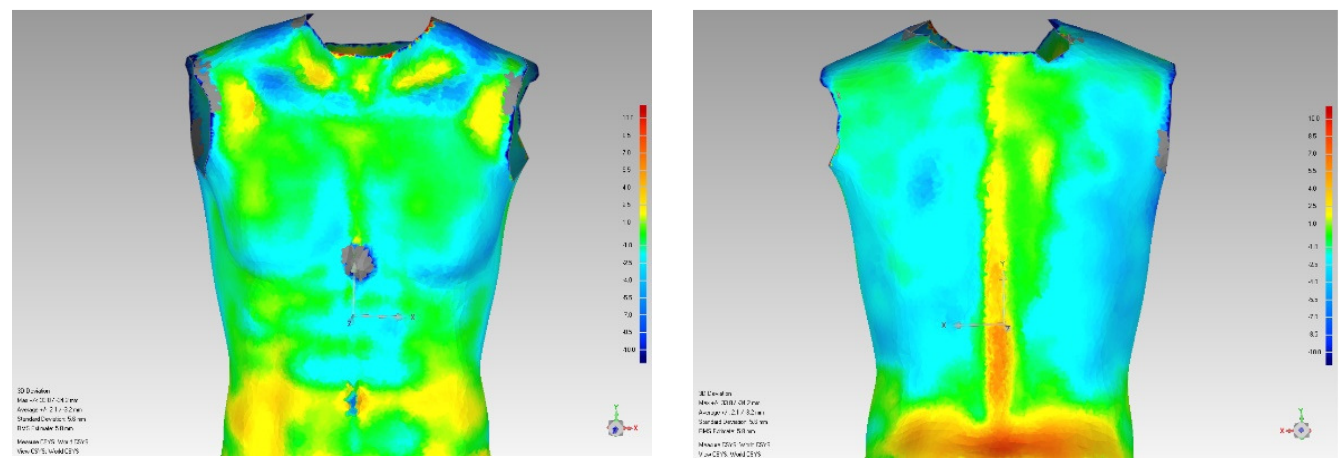

Fig. 6. INBODY $3 D$ reconstruction compared with a laser reconstruction (assumed as reference) for a static mannequin. Errors in [mm] 
Summarizing:

- INBODY presents an accuracy comparable with laser systems;

- the instant acquisition of INBODY allows the generation of clean meshes, while meshes reconstructed with some laser scanner may present overlapping problems if the patient moves during the acquisition;

- manual laser scanners require expert operators in their use, and often more passages to obtain a closed surface. In these tests, the acquisition phase of the manual laser lasted 10 minutes, and some part of the model still present holes;

- as every photogrammetric system, INBODY can reconstruct not only the geometry, but also the texture;

\section{Conclusions}

In this paper we presented INBODY, an instant photogrammetric 3D body scanner for orthopedics. We explained its hardware and software setup, as well as the developed prototype. We validated it as a possible and cost effective solution for the 3D digitization of human body models.

Several aspects need to be improved, as for example the use of workstations with more than one GPU for exploiting parallel computing. Moreover, the authors will provide INBODY of an ad-hoc software for photogrammetric processing.

The applications fields in which INBODY may be used are multiple: orthopedics, sport, medicine, fashion and beauty, cosmetic and dermatology. In the future we also expect that scanning systems may partially be used for monitoring a certain class of diseases, as scoliosis, whose diagnostics and monitoring now require invasive technologies.

\section{Acknowledgment}

The authors would like to acknowledge the people of the Ortopedia Ruggiero, in particular Francesco Bruno, for their support in testing and validating INBODY. Moreover, many thanks go to Anna Grazioso, for her graphics support.

\section{References}

[1] Chryssolouris, George, et al. "Digital manufacturing: history, perspectives, and outlook." Proceedings of the Institution of Mechanical Engineers, Part B: Journal of Engineering Manufacture 223.5 (2009): 451-462, http://dx.doi.org/10.1243/09544054JEM1241.

[2] Gibson, lan, ed. Advanced manufacturing technology for medical applications: reverse engineering, software conversion and rapid prototyping. John Wiley \& Sons, 2006, http://dx.doi.org/10.1002/0470033983.

[3] Hieu, L. C., et al. "Medical rapid prototyping applications and methods." Assembly Automation 25.4 (2005): 284-292, http://dx.doi.org/10.1108/01445150510626415

[4] ISO 8549-1:1989

[5] Baltsavias, Emmanuel P. "A comparison between photogrammetry and laser scanning." ISPRS Journal of photogrammetry and Remote Sensing 54.2 (1999): 83-94., http://dx.doi.org/10.1016/S0924-2716(99)00014-3

[6] Lowe, David G. "Object recognition from local scale-invariant features."Computer vision, 1999. The proceedings of the seventh IEEE international conference on. Vol. 2. leee, 1999., http://dx.doi.org/10.1109/ICCV.1999.790410

[7] Kazhdan, Michael, Matthew Bolitho, and Hugues Hoppe. "Poisson surface reconstruction." Proceedings of the fourth Eurographics symposium on Geometry processing. Vol. 7. 2006.

[8] Bernardini, Fausto, et al. "The ball-pivoting algorithm for surface reconstruction." IEEE transactions on visualization and computer graphics 5.4 (1999): 349-359., http://dx.doi.org/10.1109/2945.817351

[9] Hartley, Richard, and Andrew Zisserman. Multiple view geometry in computer vision. Cambridge university press, 2003.

[10] Lewandowski, Scott M. "Frameworks for component-based client/server computing." ACM Computing Survey (C s SUR) 30.1 (1998): 3-27., http://dx.doi.org/10.1145/274440.274441

[11] Kleiman, Steve, Devang Shah, and Bart Smaalders. Programming with threads. Sun Soft Press, 1996.

[12] http://www.agisoft.com/

[13] https://www.virtalis.com/files/product-brochures/fastscan-scorpion-brochure.pdf 